Article

\title{
Synthesis, Characterization and Cytotoxicity of New Rotundic Acid Derivatives
}

\author{
Yu-Fang He ${ }^{1,2, \dagger}$, Min-Lun Nan ${ }^{2, \dagger}$, Jia-Ming Sun ${ }^{3}$, Zhao-Jie Meng ${ }^{4}$, Fa-Gui Yue ${ }^{1}$, \\ Quan-Cheng Zhao ${ }^{2}$, Xiao-Hong Yang ${ }^{1} *$ and Hui Wang ${ }^{5, *}$
}

1 School of Pharmaceutical Sciences, Jilin University, Changchun 130021, China; E-Mails: he_yufang1992@163.com (Y.-F.H.); agui_228@126.com (F.-G.Y.)

2 Jilin Academy of Chinese Medicine Sciences, Changchun 130012, China; E-Mails: nanminlun2000@163.com (M.-L.N.); zhaoquancheng1954@126.com (Q.-C.Z.)

3 Development Center of Traditional Chinese Medicine and Bioengineering, Changchun University of Chinese Medicine, Changchun 130117, China; E-Mail: sun_jiaming2008@163.com

4 Norman Bethune College of Medicine, Jilin University, Changchun 130021, China;

E-Mail: mengzhaojie5555@163.com

5 China-Japan Union Hospital, Jilin University, Changchun 130033, China

$\dagger$ These authors contributed equally to this work.

* Authors to whom correspondence should be addressed;

E-Mails: yang_xiaohong88@126.com (X.-H.Y.); wanghui_1962@126.com (H.W.); Tel.: +86-431-8605-8683 (X.-H.Y.); Fax: +86-431-8605-8672 (X.-H.Y.).

Received: 5 December 2011; in revised form: 17 January 2012 / Accepted: 20 January 2012 /

Published: 31 January 2012

\begin{abstract}
Rotundic acid (RA, 1), a natural compound, exhibits potent tumor cell growth inhibiting properties. To date there are no reports on derivatives of RA. Furthermore, the 28-COOH position of RA might make it unstable and induced serious gastrointestinal side effects when it was applied in vivo. Therefore, in order to explore and make use of this compound, eight new amino acid derivatives of RA at the $28-\mathrm{COOH}$ position were synthesized and evaluated for their cytotoxicities in vitro on three tumor cell lines including A375, HepG2 and NCI-H446. As a result, a few of these new amino acid derivatives showed stronger cytotoxicity. Compound 5a was found to have the best inhibition activity on the three tested human tumor cell lines with $\mathrm{IC}_{50}$ values of less than $10 \mu \mathrm{M}$ compared with RA treatment. Meanwhile, the cytotoxicity of compound $\mathbf{6 b}$ was significantly higher than that of RA on the A375 cell line and almost the same as RA on
\end{abstract}


the HepG2 and NCI-H446 cell lines. Hence, compounds 5a and $\mathbf{6 b}$ may serve as potential lead compounds for the development of new anti-tumor drugs.

Keywords: rotundic acid; amino acid derivative; synthesis; characterization; cytotoxicity

\section{Introduction}

Rotundic acid (RA, 1, Figure 1) belongs to the pentacyclic triterpenoid family and is mainly found in Ilex rotunda, Ilex purpurea, Ilex integra and other Aquifoliaceae plants which are widely distributed in China [1-4]. RA was also isolated from Mussaenda Pubescens and Guettarda platypoda of the Rubiaceae family [5,6]. Olea europaea and Planchonella duclitan, which are part of the Oleaceae and Sapotaceae families, respectively, also contain RA [7,8]. Although there are sufficient sources for extraction of RA in China, as mentioned above, there are still few reports on its bioactivity because of little interest from pharmacological researchers. In our open patent, a considerable amount of RA was isolated and purified from I. rotunda [9]. Moreover, $\mathrm{Xu}$ et al. demonstrated that $\mathbf{R A}$, as one of many isolated compounds, showed anti-cancer activity [10]. Li et al. also reported that RA showed cytotoxicity, with $\mathrm{IC}_{50}$ values of $21.8 \mu \mathrm{M}$ and $9.5 \mu \mathrm{M}$ when it was applied on the HT29 and MCF-7 cell lines, respectively [8]. However, they did not continue to pay much attention to this compound. Since RA might be a potential native anticancer drug with sufficient sources, our research group has investigated and applied for a series of patents regarding RA and its derivatives during the past few years to explore and make use of this compound [11-15].

Figure 1. Structure of rotundic acid (RA).

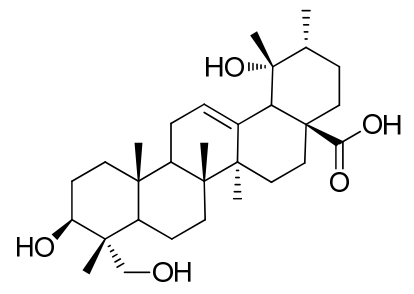

It has been widely reported that compounds with free carboxylic acids might be unstable during metabolic processes and further induce serious gastrointestinal side effect in humans. Although RA presented potential anti-tumor activity, RA with its free carboxylic acid might have the same problems when administrated in vivo [16]. Currently, structure modification is considered to be an effective method to produce lead compounds to enhance the activity and avoid possible side effects. Moreover, the structure of RA is comparatively simple, with a few active positions available for modification. These chemical modifications could be controlled easily, which would make it possible to explore new compounds with better anti-tumor activities. In this work, we carried out structure modification at the 28-COOH position of RA to improve the bioactivity of RA according to the theory of medicinal chemistry and with the experience of structural modification of pentacyclic triterpenoids [17-20]. 
Amino acids, as the basis of all metabolic cycles, are the essential compounds responsible for all life. There is a sizeable amount of literature that shows that tumor cells require larger quantities of amino acids than normal cells in the body [21,22]. Hence, in theory the selectivity of a drug for tumor cells may improve when amino acids are introduced into the drug's molecular structure. Many researchers have given much attention to the investigation of the bioactivities of amino acid drugs. Recently, many anti-tumor drugs have exhibited increased selectivity of tumor cells after undergoing amino acid modification. Their anti-tumor activities have been markedly improved and the toxicity on normal cells was lowered [23-25].

To the best of our knowledge, there are few reports on the bioactivity of RA and no reports on its derivatives. Therefore, the objective of our present study was to investigate the synthesis, characterization, and cytotoxicity of some new RA derivatives produced via introduction of amino acid groups. Their structures were elucidated on the basis of spectroscopic assays such as IR, MS, ${ }^{1} \mathrm{H}-\mathrm{NMR}$ and ${ }^{13} \mathrm{C}$-NMR. The MTT assay was employed to screen their cytotoxicity on the A375, HepG2 and NCI-H446 human cell lines.

\section{Results and Discussion}

\subsection{Preparation of $\mathbf{R A}$}

The procedure reported by $\mathrm{Xu}$ et al., for isolating $\mathbf{R A}$ from I. rotunda [10] was followed. Briefly, the barks of $I$. rotunda were shade-dried, ground, and extracted with refluxing $80 \% \mathrm{EtOH}$. The EtOH extract was evaporated ubder vacuum to obtain the total saponins fraction. The air-dried and powdered total saponins were hydrolyzed by $4 \% \mathrm{NaOH}$ in $30 \% \mathrm{EtOH}$ and purified by recrystallization to prepare RA. The purity of RA used was $\geq 98 \%$ (HPLC assay). The extraction yield of RA in our study was much higher, up to $100 \mathrm{mg} / \mathrm{g}$, which made it suitable for industry production.

\subsection{Structure Modification of $\mathbf{R A}$}

In the present study, the synthetic routes to the RA amino acid derivatives are outlined in Scheme 1. Firstly, RA (1) was converted to its 3,23-O-diacetate 2, which was then treated with oxalyl chloride to give the 28-acyl chloride 3. This intermediate was then reacted with the appropriate amino methyl ester hydrochloridea (glycine methyl ester hydrochloride, L-serine methyl ester hydrochloride, L-tryptophan methyl ester hydrochloride, L-phenylalanine methyl ester hydrochloride) in the presence

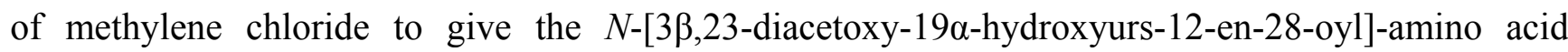
methyl esters 4a-7a. Hydrolysis of compounds 4a-7a gave the corresponding $N$ - $[3 \beta, 19 \alpha, 23-$ trihydroxyurs-12-en-28-oyl]-amino acids $\mathbf{4 b}-\mathbf{7 b}$. The structures of these synthesized compounds were confirmed by infrared (IR), mass spectra (MS), ${ }^{1} \mathrm{H}-\mathrm{NMR}$ and ${ }^{13} \mathrm{C}-\mathrm{NMR}$ [26-29]. All eight compounds obtained here were synthesized in high yields with purities of $98 \%$ or better and are reported for the first time.

It has been broadly reported that amino acid modification could enhance the anticancer activities of original compounds. Zhuo et al. demonstrated that amino acid derivatives of 5-fluorouracil had higher anti-tumor activity with lower toxicity; some of them reached $90 \%$ inhibition rate in Ehrlich carcinoma or sarcoma in mice [30-34]. Sun et al. designed and synthesized a series of amino acid conjugates of 
3-oxooleanolic acid, and determined their anti-tumor activities in vitro. Preliminary anti-tumor bioassayd showed that conjugates with higher water solubility retained anti-tumor activity [35]. In the present study, we modified the 28-COOH position of RA whereby eight new compounds were obtained. Since the amino acid modification might enhance the antitumor activities of original compound as reported, the pharmacological activity of RA and its eight derivatives were tested in the following study.

Scheme 1. Synthesis of RA derivatives.

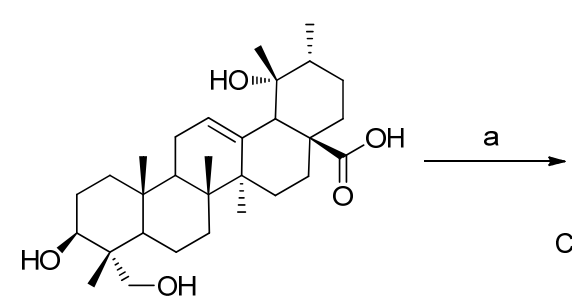

1

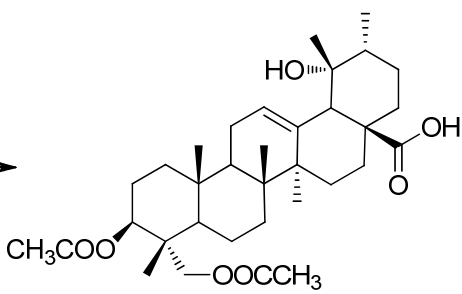

2

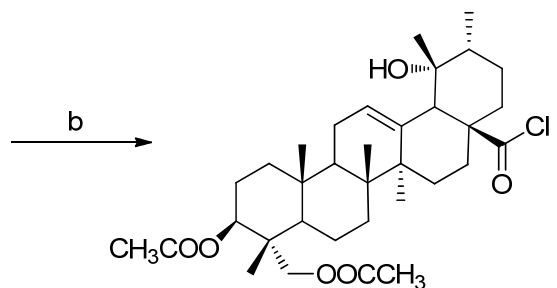

3

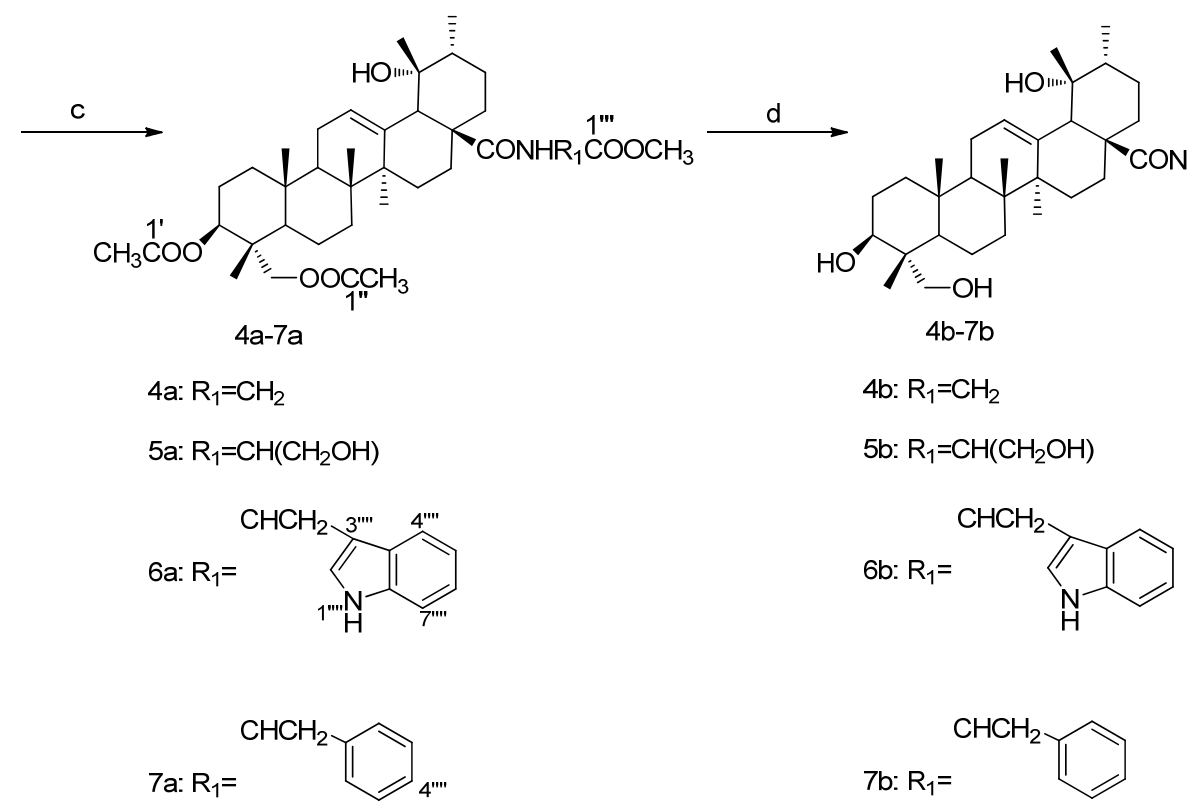

Reagents and conditions: (a) pyridine/acetic anhydride $/ 80{ }^{\circ} \mathrm{C} / 16 \mathrm{~h}$; (b) $\mathrm{CH}_{2} \mathrm{Cl}_{2} /$ oxalyl chloride/rt/20 h; (c) $\mathrm{CH}_{2} \mathrm{Cl}_{2} /$ oxalyl chloride/room temperature/24 h; $\mathrm{NH}_{2}-\mathrm{R}_{1} \mathrm{CO}_{2} \mathrm{CH}_{3} / \mathrm{Et}_{3} \mathrm{~N} / \mathrm{rt} / 12 \mathrm{~h}$; (d) $4 \% \mathrm{NaOH} / 60 \%$ methanol/reflux/6-8 h.

\subsection{Biological Activity}

In the present study, three types of human cancer cell lines including A375 (human malignant melanoma cells), HepG2 (human hepatoma cells) and NCI-H446 (human small cell lung cancer) were used to observe the cytotoxicity of RA (as a positive control) and its derivatives $4 \mathbf{a}-7 \mathbf{a}, \mathbf{4 b}-\mathbf{7 b}$. Antiproliferative effects were determined with the MTT assay [36]. Each experiment was repeated at least three times. The results are shown in Table 1 and Figure 2. 
Table 1. The $\mathrm{IC}_{50}$ values of RA and its derivatives $4 \mathbf{a}-\mathbf{7 a}, \mathbf{4 b}-\mathbf{7 b}$ on human cancer cell lines $(\mu \mathrm{M})$.

\begin{tabular}{|c|c|c|c|c|}
\hline \multirow{2}{*}{ Compound } & \multirow{2}{*}{ R1 } & \multicolumn{3}{|c|}{$\mathrm{IC}_{50} \pm \mathrm{SD}(\boldsymbol{\mu M})$} \\
\hline & & A375 & HepG2 & NCI-H446 \\
\hline $\mathbf{R A}$ & - & $16.58 \pm 1.22$ & $7.33 \pm 0.68$ & $11.40 \pm 2.32$ \\
\hline $4 \mathbf{a}$ & $\mathrm{CH}_{2}$ & $27.97 \pm 2.55$ & $10.73 \pm 1.69$ & $14.79 \pm 3.10$ \\
\hline $5 \mathbf{a}$ & $\underset{\mathrm{CHCH}_{2}}{\mathrm{CH}}\left(\mathrm{CH}_{2} \mathrm{OH}\right)$ & $5.99 \pm 0.88 *$ & $3.41 \pm 1.89 *$ & $3.84 \pm 0.12 *$ \\
\hline $6 a$ & & $20.60 \pm 0.67$ & $44.39 \pm 2.87$ & $41.78 \pm 2.36$ \\
\hline $7 \mathbf{a}$ & & $23.12 \pm 1.23$ & $85.70 \pm 3.55$ & $20.84 \pm 3.69$ \\
\hline $4 b$ & $\mathrm{CH}_{2}$ & $>100^{a}$ & $46.67 \pm 3.98$ & $15.24 \pm 1.58$ \\
\hline $5 b$ & $\underset{\mathrm{CHCH}_{2}}{\mathrm{CH}}\left(\mathrm{CH}_{2} \mathrm{OH}\right)$ & $>100^{a}$ & $22.28 \pm 2.25$ & $82.79 \pm 2.98$ \\
\hline $6 b$ & & $8.03 \pm 0.87 *$ & $6.11 \pm 1.00$ & $11.32 \pm 1.56$ \\
\hline $7 b$ & & $34.59 \pm 1.96$ & $14.19 \pm 0.98$ & $11.99 \pm 1.48$ \\
\hline
\end{tabular}

Notes: Data are represented in mean $\pm \mathrm{SD} ; n=3{ }^{\mathrm{a}} \mathrm{IC}_{50}$ values more than $100 \mu \mathrm{M}$ are indicated as $>100,{ }^{*} p<0.05 v s$. RA.

Figure 2. Inhibitory effect of $\mathbf{5 a}$ on the human cancer cell proliferation. (A) A375; (B) HepG2; (C) NCI-H446; (D) $\mathrm{IC}_{50}$ of RA and compound 5a, ${ }^{*} p<0.05$ vs. RA.

A
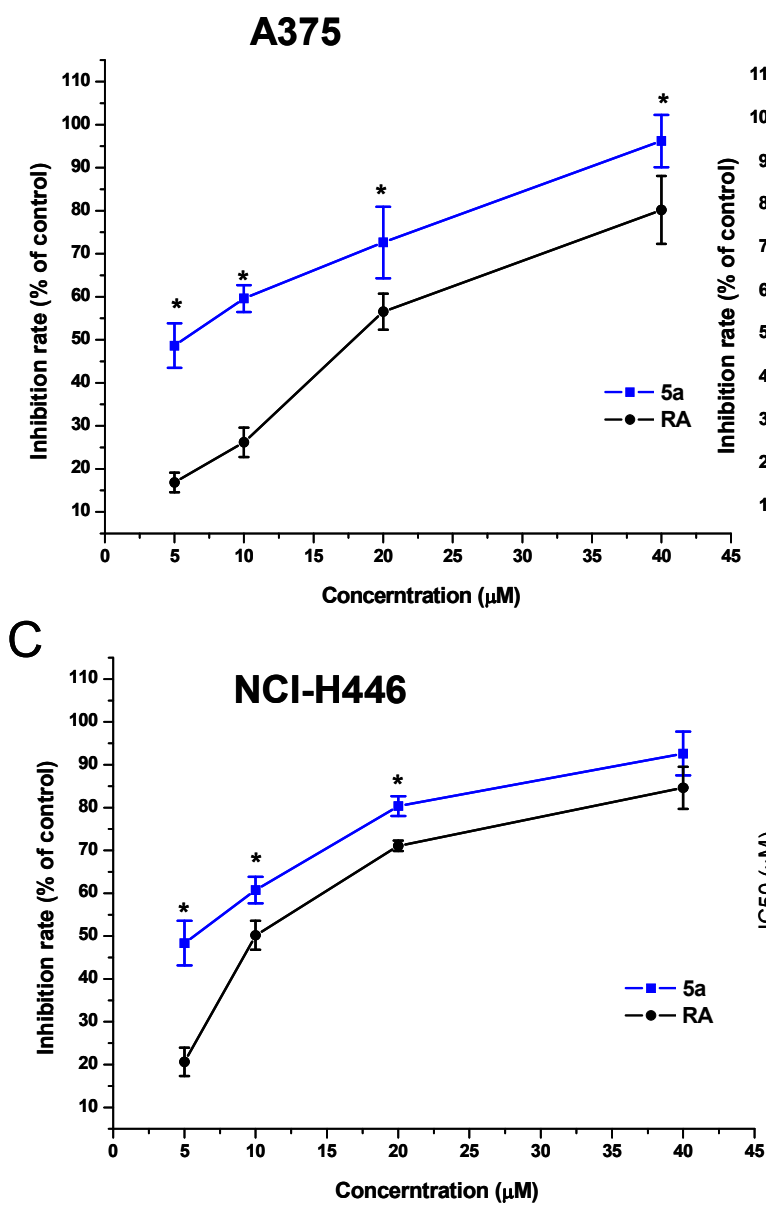

B

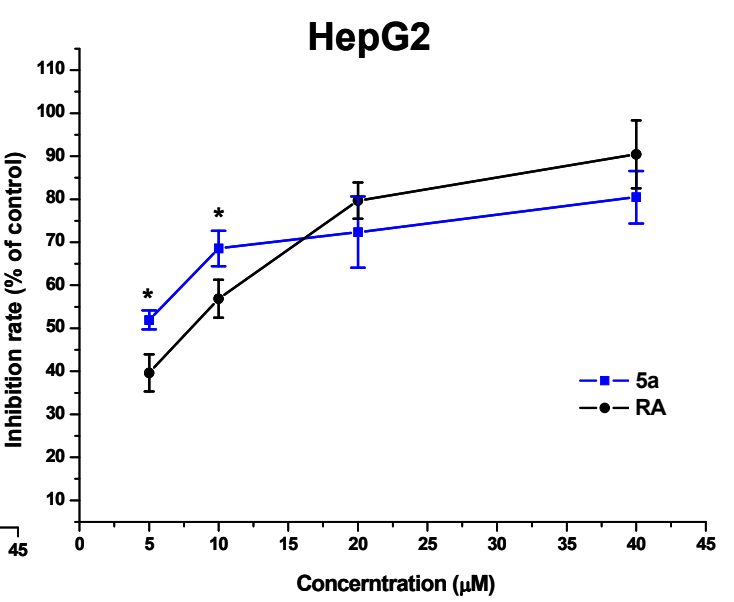

D

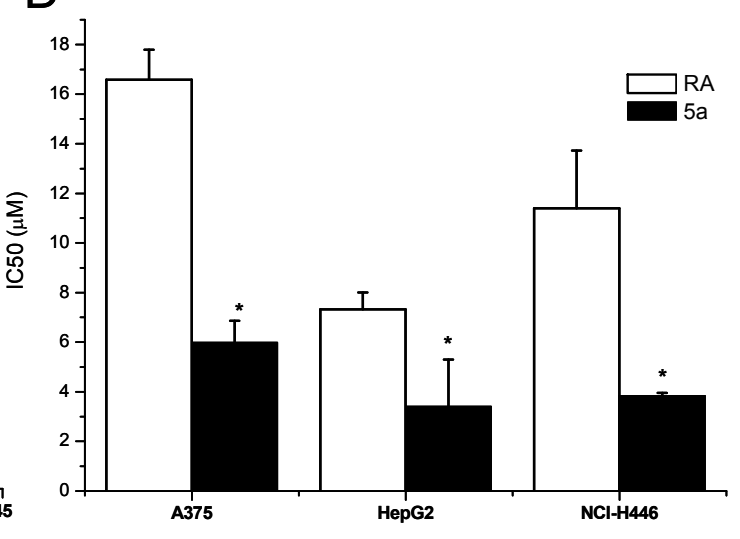


As shown in Table 1, RA showed significant $\mathrm{IC}_{50}$ values of $16.58,7.33,11.40 \mu \mathrm{M}$ on $\mathrm{A} 375$, HepG2, and NCI-H446, respectively, which is consistent with the previous results by Li et al. [8] and $\mathrm{Xu}$ et al. [10], who investigated the cytotoxicity of RA on HeLa, MDA-MB-435, CNE1, HT29b, MCF-7c etc. Based on the cytotoxicity of RA, we tested the cytotoxicity of the compounds $\mathbf{4 a - 7 a , ~} \mathbf{4 b}-\mathbf{7 b}$. The result demonstrated that compounds $4 \mathbf{a}$ and $5 \mathbf{a}$ presented more potent anti-tumor activity on the A375, HepG2 and NCI-H446 cell lines compared to compounds $\mathbf{4 b}$ and $\mathbf{5 b}$. Interestingly, when comparing compounds $\mathbf{6 a}$ and $7 \mathbf{a}$ to compounds $\mathbf{6 b}$ and $\mathbf{7 b}$, the results were just the opposite. The compounds $\mathbf{6 b}$ and $\mathbf{7 b}$ presented more potent anti-tumor activity on the A375, HepG2 and NCI-H446 cell lines compared to compounds $\mathbf{6 a}$ and 7a (Table 1). The results might be explained by a steric hindrance arising from the conjugation of an amino group at C-28. When the group is an amino acid methyl ester, the activity of a small steric group is more potent than that of a large one, but when the group is an amino acid, a large steric hindrance is more effective than a small one. In addition, we can see from Table 1 that the cytotoxicity of $\mathbf{6 b}$ was similar to that of the RA treatment on the HepG2 and NCI-H446 cell lines, and significantly higher than the RA treatment on the A375 cell line $(8.03 \mu \mathrm{M} v s$. $16.58 \mu \mathrm{M})$. Also, the $\mathrm{IC}_{50}$ of $\mathbf{6 b}$ on HepG2 was also less than $10 \mu \mathrm{M}(6.11 \mu \mathrm{M})$, but it was not significantlt different when compared to the $\mathrm{IC}_{50}$ of $\mathbf{R A}$. These results demonstrate that compound $\mathbf{6 b}$ might be a potential anticancer drug and this will require further investigation. Furthermore, as shown in Figure 2, the inhibitory rates of compound 5a on the three cell lines were significantly increased at low concentration $(5.0$ and $10.0 \mu \mathrm{M})$ compared to $\mathbf{R A}$ treatment in a dose dependent manner. The $\mathrm{IC}_{50}$ of compound 5a was significantly less than RA treatment group $(<10 \mu \mathrm{M})$, which indicated that compound 5a could be a candidate for the development of new anticancer drugs. It was well known that hydroxyl groups can increase water solubility which might enhance the activity of the compound (the $\mathrm{IC}_{50}$ values of compound $\mathbf{5 a}$ on the three cell lines were 5.99, 3.41, and $3.84 \mu \mathrm{M}$, respectively). However, further investigation of compound 5a still needs to be conducted and could include studying its anticancer activity in vivo.

\section{Experimental}

\subsection{General}

Reagent-grade chemicals and solvents were obtained from commercial suppliers. Melting points were determined on a Fisher-Johns apparatus and are uncorrected. IR spectra were recorded on a Perkin-Elmer $983 \mathrm{G}$ spectrometer. NMR spectra were measured in $\mathrm{C}_{5} \mathrm{D}_{5} \mathrm{~N}$ on a Bruker AM-400 spectrometer, using TMS as an internal standard. NMR experiments included the HMQC and HMBC pulse sequences. Coupling constants ( $J$ values) are given in Hz, and a MS Agilent 1100 Series LC/MSD ion-trap mass spectrometer was used to record the ESI-MS and HR-ESI-MS. All solvents were freshly distilled and dried prior to use, according to the standard procedures. All chemicals were purchased from Sigma Chemicals Ltd. The human hematoma cell line (HepG2), human malignant melanoma cell line (A375) and human small cell lung cancer cell line (NCI-H446) were purchased from Jilin Provincial Tumor Hospital. 


\subsection{Extraction and Isolation of RA (1)}

The barks $(1.0 \mathrm{~kg})$ of $I$. rotunda were shade-dried, ground, and extracted with refluxing $80 \% \mathrm{EtOH}$ successively ( $8 \mathrm{~L}, 3 \mathrm{~h}$, two times). The EtOH extract was evaporated in vacuo to yield total saponins $(100 \mathrm{~g})$. The air-dried and powdered total saponins (100.0 g) were refluxed with $4 \% \mathrm{NaOH}$ in $30 \%$ $\operatorname{EtOH}(5.0 \mathrm{~L})$ at $100{ }^{\circ} \mathrm{C}$ for $4 \mathrm{~h}$. The mixture was then cooled to room temperature and extracted with EtOAc $(1.0 \mathrm{~L} \times 3)$. The combined organic layers were concentrated under reduced pressure to give the residue $\left(47.1 \mathrm{~g}\right.$ ), which was recrystallized by $\mathrm{MeOH}-\mathrm{H}_{2} \mathrm{O}$ to yield pure $\mathbf{R A}(32.3 \mathrm{~g}) . \mathrm{mp} 272.0-273.5^{\circ} \mathrm{C}$; IR (KBr) cm ${ }^{-1}: 3570,3417,2933,2878,1689,1460,1388,1046$ and 933; ${ }^{1} \mathrm{H}-\mathrm{NMR} \delta: 5.50$ (1H, m, H-12), $4.84(1 \mathrm{H}, \mathrm{m}, \mathrm{H}-3), 4.07$ (1H, m, H-23a), $3.60(1 \mathrm{H}, \mathrm{d}, J=10.3 \mathrm{~Hz}, \mathrm{H}-23 \mathrm{~b}), 2.97$ (1H, br s, H-18), $1.57\left(3 \mathrm{H}, \mathrm{s}, \mathrm{CH}_{3}-25\right), 1.32\left(3 \mathrm{H}, \mathrm{s}, \mathrm{CH}_{3}-27\right), 1.02\left(3 \mathrm{H}, \mathrm{s}, \mathrm{CH}_{3}-29\right), 1.00\left(3 \mathrm{H}, \mathrm{d}, J=6.64 \mathrm{~Hz}, \mathrm{CH}_{3}-30\right)$, $0.95\left(3 \mathrm{H}, \mathrm{s}, \mathrm{CH}_{3}-26\right), 0.88\left(3 \mathrm{H}, \mathrm{s}, \mathrm{CH}_{3}-24\right) ;{ }^{13} \mathrm{C}-\mathrm{NMR} \delta: 180.8$ (C-28), 140.1 (C-13), 128.2 (C-12), 73.7 (C-3), 72.8 (C-19), 68.2 (C-23), 54.8 (C-18), 48.8 (C-5), 48.4 (C-9), 47.9 (C-17), 43.0 (C-20), 42.5 (C-14), 42.3 (C-8), 40.5 (C-1), 39.0 (C-4), 38.6 (C-22), 37.3 (C-10), 33.4 (C-7), 29.5 (C-15), 27.8 (C-21), 27.2 (C-29), 27.0 (C-2), 26.5 (C-16), 24.8 (C-27), 24.2 (C-11), 18.9 (C-6), 17.4 (C-25), 16.9 (C-26), 16.1 (C-30), 13.2 (C-24). ESI-MS m/z: $489.4[\mathrm{M}+\mathrm{H}]^{+}$.

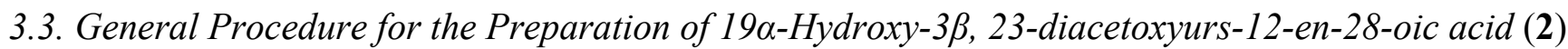

RA $(1.02 \mathrm{mmol})$ was dissolved in pyridine $(20 \mathrm{~mL})$, then acetic anhydride $(10 \mathrm{~mL})$ was added to mixture which was stirred at $80{ }^{\circ} \mathrm{C}$ for $16 \mathrm{~h}$. The solvent was removed under reduced pressure using a rotary evaporator. The residue was washed with water, and evaporated to dryness. The residue was purified by column chromatography on silica gel to give compound $\mathbf{2}$ as colorless needles. Yield, 69.9\%; mp 156.5-158.5 ${ }^{\circ} \mathrm{C}$; IR (KBr) cm ${ }^{-1}: 3597,3443,2955,2873,1727,1704,1472,1370,1036$ and 924; ${ }^{1} \mathrm{H}-\mathrm{NMR} \delta: 5.47$ (1H, m, H-12), 4.97 (1H, m, H-3), 4.89 (1H, m, H-23a), 3.89 (1H, brs, H-23b), $2.93\left(1 \mathrm{H}\right.$, br s, H-18), $1.91\left(3 \mathrm{H}, \mathrm{s}, \mathrm{CH}_{3}-1^{\prime}\right), 1.86\left(3 \mathrm{H}, \mathrm{s}, \mathrm{CH}_{3}-1 "\right), 1.63\left(3 \mathrm{H}, \mathrm{s}, \mathrm{CH}_{3}-25\right), 1.32(3 \mathrm{H}, \mathrm{s}$, $\left.\mathrm{CH}_{3}-27\right), 0.97$ (3H, s, $\left.\mathrm{CH}_{3}-29\right), 1.00\left(3 \mathrm{H}, \mathrm{d}, J=6.64 \mathrm{~Hz}, \mathrm{CH}_{3}-30\right), 0.76\left(3 \mathrm{H}, \mathrm{s}, \mathrm{CH}_{3}-26\right), 0.71(3 \mathrm{H}, \mathrm{s}$, $\left.\mathrm{CH}_{3}-24\right) ;{ }^{13} \mathrm{C}-\mathrm{NMR} \delta$ : 180.8 (C-28), 140.1 (C-13), 127.9 (C-12), 74.8 (C-3), 72.8 (C-19), 66.8 (C-23), 54.7 (C-18), 48.5 (C-5), 48.4 (C-9), 47.9 (C-17), 42.5 (C-20), 42.2 (C-14), 41.0 (C-8), 40.4 (C-1), 38.6 (C-4), 38.0 (C-22), 37.0 (C-10), 33.2 (C-7), 29.4 (C-15), 27.2 (C-21), 27.0 (C-29), 26.5 (C-2), 24.6 (C-16), 24.0 (C-27), 23.5 (C-11), 18.5 (C-6), 17.3 (C-25), 16.9 (C-26), 16.0 (C-30), 13.3 (C-24), 170.7 (C-1'), 21.2 (C-2'), 170.6 (C-1"), 20.8 (C-2"). ESI-MS m/z: $573.1[\mathrm{M}+\mathrm{H}]^{+}$. HR-ESI-MS found: 573.3804. calcd: 573.3791 for $\mathrm{C}_{34} \mathrm{H}_{53} \mathrm{O}_{7}\left([\mathrm{M}+\mathrm{H}]^{+}\right)$.

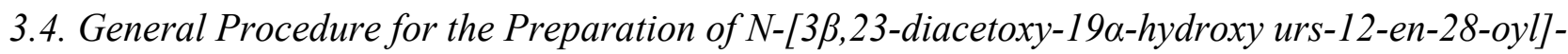 amino acid methyl esters $\mathbf{4 a - 7 a}$}

To a solution of compound $2(2.33 \mathrm{mmol})$ in $\mathrm{CH}_{2} \mathrm{Cl}_{2}(25 \mathrm{~mL})$ added oxalyl chloride $(2 \mathrm{~mL})$ and the mixture was stirred at an ice-water bath for $1 \mathrm{~h}$, then further stirred at room temperature for $24 \mathrm{~h}$. The mixture was concentrated to dryness under reduced pressure $\left(30^{\circ} \mathrm{C}\right) . \mathrm{CH}_{2} \mathrm{Cl}_{2}$ was added to the residue three times (each time $50 \mathrm{~mL}$ ), then the concentrated to dryness to yield crude 3,23-O-diacetylursolyl chloride 3. Next in an ice-water bath, glycine methyl ester hydrochloride (12 mmol, which was dissolved in $60 \mathrm{~mL} \mathrm{CH}_{2} \mathrm{Cl}_{2}$ and $6 \mathrm{~mL}$ triethylamine) was added to a $\mathrm{CH}_{2} \mathrm{Cl}_{2}$ 
solution $(90 \mathrm{~mL})$ of $\mathbf{3}(2.33 \mathrm{mmol})$ The reaction mixture was stirred in the ice-water bath for $0.5 \mathrm{~h}$, and then stirred at room temperature for $24 \mathrm{~h}$, and then washed in turn with $2.5 \%$ hydrochloric acid, water, and saturated sodium chloride solution (each liquid three times, each time $50 \mathrm{~mL}$ ). Then the reaction mixture was treated with anhydrous sodium sulfate, filtered, concentrated, and then dried to yield a light yellow solid that was recrystallized from $95 \%$ ethanol $(400 \mathrm{~mL})$ to yield a white solid. The solid was purified on a silica gel column with petroleum ether and ethyl acetate as eluents to yield white needles.

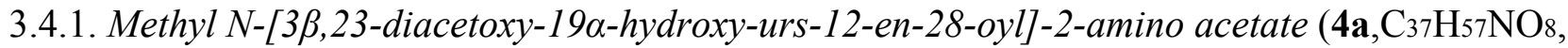
$\left.\mathrm{R}_{1}=\mathrm{CH}_{2}\right)$

Yield 56.9\%, mp $198 \sim 200{ }^{\circ} \mathrm{C}$; IR (KBr) cm ${ }^{-1}: 3411,2972,2925,2882,1741,1727,1651,1520$, 1474, 1444, 1386, 1370, 1250, 1049, 1028 and 1004; ${ }^{1} \mathrm{H}-\mathrm{NMR} \delta: 8.07$ (1H, brs, -NH), 5.44 (1H, m, H-12), $4.93(1 \mathrm{H}, \mathrm{m}, \mathrm{H}-3), 4.25$ (1H, m, H-2'" a), 4.10 (1H, m, H-2'" b), 3.93 (1H, d, J = $11.6 \mathrm{~Hz}, \mathrm{H}-23 \mathrm{a})$, $3.87(1 \mathrm{H}, \mathrm{d}, J=11.6 \mathrm{~Hz}, \mathrm{H}-23 \mathrm{~b}), 3.51\left(3 \mathrm{H}, \mathrm{s}, 1{ }^{\prime \prime}-\mathrm{OCH}_{3}\right), 2.82\left(1 \mathrm{H}\right.$, br s, H-18), $1.91\left(3 \mathrm{H}, \mathrm{s}, \mathrm{CH}_{3}-2^{\prime}\right)$, $1.86\left(3 \mathrm{H}, \mathrm{s}, \mathrm{CH}_{3}-2 "\right), 1.60\left(3 \mathrm{H}, \mathrm{s}, \mathrm{CH}_{3}-25\right), 1.27\left(3 \mathrm{H}, \mathrm{s}, \mathrm{CH}_{3}-27\right), 0.87\left(3 \mathrm{H}, \mathrm{s}, \mathrm{CH}_{3}-29\right), 0.95(3 \mathrm{H}, \mathrm{d}$, $\left.J=6.64 \mathrm{~Hz}, \mathrm{CH}_{3}-30\right), 0.82\left(3 \mathrm{H}, \mathrm{s}, \mathrm{CH}_{3}-26\right), 0.74\left(3 \mathrm{H}, \mathrm{s}, \mathrm{CH}_{3}-24\right) ;{ }^{13} \mathrm{C}-\mathrm{NMR} \delta: 178.7(\mathrm{C}-28)$, 140.0 (C-13), 128.1 (C-12), 74.8 (C-3), 73.1 (C-19), 65.7 (C-23), 54.4 (C-18), 48.5 (C-5), 48.1 (C-9), 47.9 (C-17), 42.3 (C-20), 42.1 (C-14), 41.0 (C-8), 40.4 (C-1), 38.9 (C-4), 38.0 (C-22), 37.1 (C-10), 33.2 (C-7), 28.9 (C-15), 27.2 (C-21), 27.1 (C-29), 26.2 (C-2), 24.6 (C-16), 24.0 (C-27), 23.5 (C-11), 18.5 (C-6), 17.1 (C-25), 16.9 (C-26), 16.0 (C-30), 13.3 (C-24), 170.7 (C-1'), 21.2 (C-2'), 170.5 (C-1"), 20.8 (C-2"), 171.6 (C-1"'), 51.8 (1"'-OCH $\left.{ }_{3}\right), 42.0$ (C-2"'). ESI-MS m/z: 644.4 [M+H] ${ }^{+}$. HR-ESI-MS found: 644.4122. calcd: 644.4157 for $\mathrm{C}_{37} \mathrm{H}_{58} \mathrm{NO}_{8}\left([\mathrm{M}+\mathrm{H}]^{+}\right)$.

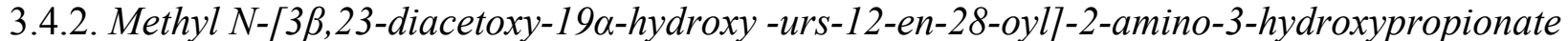

(5a, $\mathrm{C}_{38} \mathrm{H}_{59} \mathrm{NO}_{9}, \mathrm{R}_{1}=\mathrm{CH}\left(\mathrm{CH}_{2} \mathrm{OH}\right)$

Compound 2 was reacted with L-serine methyl ester using general procedure to give compound $\mathbf{5 a}$. Eluted with petroleum ether/ethyl acetate $(\mathrm{V} / \mathrm{V})=5: 5$. Colorless white needles, yield $64.2 \%$, mp $143 \sim 145^{\circ} \mathrm{C}$; IR (KBr) cm ${ }^{-1}: 3443,2935,2879,1744,1653,1509,1470,1371,1248,1035$ and 935; ${ }^{1} \mathrm{H}-\mathrm{NMR}$ 8: 7.42 (1H, brs, -NH), 5.50 (1H, m, H-12), 5.03 (1H, m, H-2"'), 4.93 (1H, m, H-3), 4.25 (2H, m, H-3"'), 3.90 (1H, br s, H-23), 3.59 (3H, s, 1"'-OCH $), 2.75$ (1H, br s, H-18), 1.92 (3H, s, $\left.\mathrm{CH}_{3}-2^{\prime}\right)$, $1.85\left(3 \mathrm{H}, \mathrm{s}, \mathrm{CH}_{3}-2 "\right), 1.59\left(3 \mathrm{H}, \mathrm{s}, \mathrm{CH}_{3}-25\right), 1.25\left(3 \mathrm{H}, \mathrm{s}, \mathrm{CH}_{3}-27\right), 0.93\left(3 \mathrm{H}, \mathrm{d}, J=6.64 \mathrm{~Hz}, \mathrm{CH}_{3}-30\right)$, $0.85\left(3 \mathrm{H}, \mathrm{s}, \mathrm{CH}_{3}-29\right), 0.80$ (3H, s, $\left.\mathrm{CH}_{3}-26\right), 0.74$ (3H, s, $\left.\mathrm{CH}_{3}-24\right) ;{ }^{13} \mathrm{C}-\mathrm{NMR} \delta: 178.2$ (C-28), 139.7 (C-13), 128.5 (C-12), 74.7 (C-3), 73.0 (C-19), 65.7 (C-23), 54.6 (C-18), 48.5 (C-5), 48.1 (C-9), 47.9 (C-17), 42.2 (C-20), 42.1 (C-14), 41.0 (C-8), 40.5 (C-1), 38.9 (C-4), 38.0 (C-22), 37.1 (C-10), 33.2 (C-7), 28.9 (C-15), 27.1 (C-21), 27.0 (C-29), 26.2 (C-2), 24.5 (C-16), 24.1(C-27), 23.5 (C-11), 18.5 (C-6), 17.1 (C-25), 16.8 (C-26), 16.0 (C-30), 13.3 (C-24), 170.7 (C-1'), 21.2 (C-2'), 170.5 (C-1"), 20.8 (C-2"), 172.6 (C-1'"), 62.9 (C-3"'), 56.2 (C-2"'), 52.2 (1"'-OCH $\left.{ }_{3}\right)$. ESI-MS m/z: 674.5 [M+H] $]^{+}$HR-ESI-MS found: 674.4222. calcd: 674.4263 for $\mathrm{C}_{38} \mathrm{H}_{60} \mathrm{NO}_{9}\left([\mathrm{M}+\mathrm{H}]^{+}\right)$. 


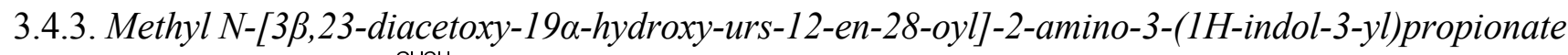
(6a, $\mathrm{C}_{46} \mathrm{H}_{64} \mathrm{~N}_{2} \mathrm{O}_{8}, \mathrm{R}_{1}=$

Compound 2 was reacted using the general procedure with L-tryptophan methyl ester to give compound 6a. Eluted with petroleum ether/ethyl acetate $(\mathrm{V} / \mathrm{V})=5: 2$. White needles, yield 54.5\%, mp $243 \sim 245{ }^{\circ} \mathrm{C}$; IR (KBr) cm ${ }^{-1}$ : 3410, 3372, 2966, 2952, 2878, 1730, 1651, 1519, 1460, 1444, 1368, 1250, 1019 and 742; ${ }^{1} \mathrm{H}-\mathrm{NMR} \delta: 11.9(1 \mathrm{H}, \mathrm{s}$, indole-NH), 7.98 (1H, brs, amide-NH), 7.48 (1H, brs, H-4"'"), 7.46 (1H, s, H-2'"'), 7.35 (1H, m, H-7'"'), 7.16 (2H, m, H-5"'" and H-6"''), 5.33 (1H, m, H-12), 5.11 (1H, m, H-2"'), 4.91 (1H, m, H-3), 3.90 (2H, m, H-23), 3.64 (3H, s, 1"'-OCH $), 3.35$ (2H, m, H-3"'), $2.77\left(1 \mathrm{H}\right.$, br s, H-18), $1.96\left(3 \mathrm{H}, \mathrm{s}, \mathrm{CH}_{3}-2^{\prime}\right), 1.85\left(3 \mathrm{H}, \mathrm{s}, \mathrm{CH}_{3}-2^{\prime \prime}\right), 1.50\left(3 \mathrm{H}, \mathrm{s}, \mathrm{CH}_{3}-25\right), 1.20(3 \mathrm{H}, \mathrm{s}$, $\left.\mathrm{CH}_{3}-27\right), 0.91\left(3 \mathrm{H}, \mathrm{d}, J=6.64 \mathrm{~Hz}, \mathrm{CH}_{3}-30\right), 0.75\left(3 \mathrm{H}, \mathrm{s}, \mathrm{CH}_{3}-29\right), 0.70$ (3H, s, $\left.\mathrm{CH}_{3}-26\right), 0.29$ (3H, s, $\left.\mathrm{CH}_{3}-24\right) ;{ }^{13} \mathrm{C}-\mathrm{NMR} \delta$ : 178.2 (C-28), 139.7 (C-13), 137.9 (C-9"'"), 128.5 (C-8"'"), 128.1 (C-12), 124.5 (C-2'"'), 122.3 (C-6"''), 119.6 (C-5"''), 119.1 (C-7"'"), 112.4 (C-4"'"), 110.9 (C-3"''), 74.8 (C-3), 73.0 (C-19), 65.8 (C-23), 54.1 (C-18), 48.4 (C-5), 47.9 (C-9), 47.8 (C-17), 42.3 (C-20), 41.9 (C-14), 41.0 (C-8), 40.1 (C-1), 38.6 (C-4), 38.0 (C-22), 37.0 (C-10), 32.8 (C-7), 28.6 (C-15), 27.2 (C-21), 27.1 (C-29), 26.2 (C-2), 24.5 (C-16), 23.9 (C-27), 23.5 (C-11), 18.6 (C-6), 16.8 (C-25), 16.2 (C-26), 15.9 (C-30), 13.4 (C-24), 170.7 (C-1'), 21.2 (C-2'), 170.6 (C-1"), 20.8 (C-2"), 174.0 (C-1"'), 28.2 (C-3"'), 54.7 (C-2"'), $52.1\left(1 " '-\mathrm{OCH}_{3}\right)$. ESI-MS m/z: $773.5[\mathrm{M}+\mathrm{H}]^{+}$. HR-ESI-MS found: 773.4715. calcd: 773.4735 for $\mathrm{C}_{46} \mathrm{H}_{65} \mathrm{~N}_{2} \mathrm{O}_{8}\left([\mathrm{M}+\mathrm{H}]^{+}\right)$.

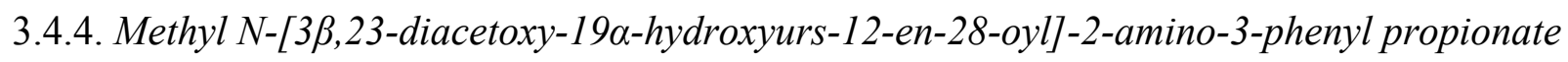
(7a, $\left.\mathrm{C}_{44} \mathrm{H}_{63} \mathrm{NO}_{8}, \mathrm{R}_{1}=\mathrm{CHCH}_{2}\right)$

Compound 2 was reacted using the general procedure with L-phenylalanine methyl ester to afford compound 7a. Eluted with petroleum ether/ethyl acetate $(\mathrm{V} / \mathrm{V})=5: 2$. White needles, yield $62.0 \%, \mathrm{mp}$ $145 \sim 147{ }^{\circ} \mathrm{C}$; IR (KBr) cm ${ }^{-1}: 3603,3455,3383,2940,2866,1753,1728,1714,1653,1518,1471$, 1444, 1379, 1252, 1037, 744 and 696; ${ }^{1} \mathrm{H}-\mathrm{NMR} \delta: 7.54$ (1H, brs, -NH), 7.28 (4H, m, H-2"'", 3"'", 5"'", and 6"''), 7.20 (1H, m, H-4'"'), 5.39 (1H, m, H-12), 5.00 (1H, m, H-2'"), 4.92 (1H, m, H-3), 3.98 (2H, m, H-23), 3.62 (3H, s, 1'"--OCH $), 3.15$ (2H, m, H-3"'), 2.75 (1H, br s, H-18), 1.92 (3H, s, $\left.\mathrm{CH}_{3}-2^{\prime}\right)$, $1.87\left(3 \mathrm{H}, \mathrm{s}, \mathrm{CH}_{3}-2\right)$ ), $1.55\left(3 \mathrm{H}, \mathrm{s}, \mathrm{CH}_{3}-25\right), 1.22\left(3 \mathrm{H}, \mathrm{s}, \mathrm{CH}_{3}-27\right), 0.93\left(3 \mathrm{H}, \mathrm{d}, J=6.64 \mathrm{~Hz}, \mathrm{CH}_{3}-30\right)$, 0.79 (3H, s, $\left.\mathrm{CH}_{3}-29\right), 0.76$ (3H, s, $\left.\mathrm{CH}_{3}-26\right), 0.54\left(3 \mathrm{H}, \mathrm{s}, \mathrm{CH}_{3}-24\right) ;{ }^{13} \mathrm{C}-\mathrm{NMR}$ $: 178.4$ (C-28), 139.7 (C-13), 138.2 (C-1"''), 129.9 (C-2"'" and 6"''), 129.1 (C-3"'" and 5"''), 128.2 (C-12), 127.3 (C-4'"'), 74.8 (C-3), 73.0 (C-19), 65.8 (C-23), 54.2 (C-18), 48.5 (C-5), 48.0 (C-9), 47.9 (C-17), 42.3 (C-20), 42.0 (C-14), 41.0 (C-8), 40.3 (C-1), 38.5 (C-4), 38.0 (C-22), 37.0 (C-10), 32.9 (C-7), 28.8 (C-15), 27.2 (C-21), 27.1 (C-29), 26.1 (C-2), 24.5 (C-16), 24.0 (C-27), 23.5 (C-11), 18.4 (C-6), 16.9 (C-25), 16.8 (C-26), 16.0 (C-30), 13.4 (C-24), 170.7 (C-1'), 21.2 (C-2'), 170.6 (C-1"), 20.8 (C-2"), 173.5 (C-1"'), 28.2 (C-3"'), 55.3 (C-2'"), 52.1 (1"'-OCH 3 ). ESI-MS m/z: 734.5 [M+H] $]^{+}$. HR-ESI-MS found: 734.4888. calcd: 734.4632 for $\mathrm{C}_{44} \mathrm{H}_{64} \mathrm{NO}_{8}\left([\mathrm{M}+\mathrm{H}]^{+}\right)$. 
3.5. General Procedure for the Preparation of $\mathrm{N}-[3 \beta, 19 \alpha, 23$-trihydroxy-urs-12-en-28-oyl] amino acids $\mathbf{4 b}-\mathbf{7 b}$

A solution of $4 \mathbf{a}$ (or 5a-7a) was stirred and refluxed for $6-8 \mathrm{~h}$ with aqueous $\mathrm{NaOH}(4 \%)$ in $60 \%$ $\mathrm{CH}_{3} \mathrm{OH}$, cooled, water $(50 \mathrm{~mL})$ was added, and then treated with $2 \mathrm{~N} \mathrm{HCl}$ to $\mathrm{pH} 5$, filtered, the solid was washed with water, and dried to give a white powder.

\subsection{1. $N$-[3 $\beta, 19 \alpha, 23$-trihydroxyurs-12-en-28-oyl]-2-amino acetic acid $\left(\mathbf{4 b}, \mathrm{C}_{32} \mathrm{H}_{51} \mathrm{NO}_{6}, \mathrm{R}_{1}=\mathrm{CH}_{2}\right)$}

Yield 83.3\%, mp $224 \sim 226{ }^{\circ} \mathrm{C}$; IR (KBr) cm ${ }^{-1}: 3451,3369,2932,2876,1634,1611,1525,1460$, 1367, 1046 and 932; ${ }^{1} \mathrm{H}-\mathrm{NMR}$ 8: 7.74 (1H, brs, -NH), 5.53 (1H, brs, H-12), 5.01 (1H, m, H-3), 4.43 (1H, m, H-1"' a), 4.28 (1H, m, H-1'" b), 4.07 (2H, m, H-23), 3.77 (2H, m, H-2'"), 2.80 (1H, br s, H-18), $1.55\left(3 \mathrm{H}, \mathrm{s}, \mathrm{CH}_{3}-25\right), 1.28\left(3 \mathrm{H}, \mathrm{s}, \mathrm{CH}_{3}-27\right), 0.97\left(3 \mathrm{H}, \mathrm{s}, \mathrm{CH}_{3}-29\right), 0.96\left(3 \mathrm{H}, \mathrm{d}, J=6.64 \mathrm{~Hz}, \mathrm{CH}_{3}-30\right)$, $0.97\left(3 \mathrm{H}, \mathrm{s}, \mathrm{CH}_{3}-26\right), 0.91\left(3 \mathrm{H}, \mathrm{s}, \mathrm{CH}_{3}-24\right) ;{ }^{13} \mathrm{C}-\mathrm{NMR} \delta: 178.6$ (C-28), 140.1 (C-13), $128.6(\mathrm{C}-12)$, 73.7 (C-3), 73.1 (C-19), 68.2 (C-23), 54.8 (C-18), 48.8 (C-5), 48.1 (C-9), 48.0 (C-17), 43.0 (C-20), 42.6 (C-14), 42.4 (C-8), 40.6 (C-1), 39.0 (C-4), 39.0 (C-22), 37.3 (C-10), 33.3 (C-7), 29.0 (C-15), 27.8 (C-21), 27.3 (C-29), 27.2 (C-2), 26.4 (C-16), 24.8 (C-27), 24.2 (C-11), 18.9 (C-6), 17.1 (C-25), 16.9 (C-26), 16.2 (C-30), 13.2 (C-24), 173.5 (C-1"'), 42.3 (C-2'"). ESI-MS m/z: 546.3 [M+H]. HR-ESI-MS found: 546.3711. calcd: 546.3795 for $\mathrm{C}_{32} \mathrm{H}_{52} \mathrm{NO}_{6}\left([\mathrm{M}+\mathrm{H}]^{+}\right)$.

3.5.2. $\mathrm{N}$-[3 $\beta, 19 \alpha, 23$-trihydroxy urs-12-en-28-oyl]-2-amino-3-hydroxypropionic acid $\left(\mathbf{5} \mathbf{b}, \mathrm{C}_{33} \mathrm{H}_{53} \mathrm{NO}_{7}\right.$, $\mathrm{R}_{1}=\mathrm{CH}\left(\mathrm{CH}_{2} \mathrm{OH}\right)$

White powder, yield 88.1\%, mp $248 \sim 249{ }^{\circ} \mathrm{C}$; IR $(\mathrm{KBr}) \mathrm{cm}^{-1}: 3412,2936,2876,1726,1631,1515$, 1466, 1388, 1350, 1037 and 938; ${ }^{1} \mathrm{H}-\mathrm{NMR} \delta: 7.43(1 \mathrm{H}$, brs, -NH), 5.60 (1H, brs, H-12), $5.15(1 \mathrm{H}, \mathrm{m}$, H-2"'), 5.11 (1H, m, H-3), 4.47 (2H, m, H-3"'), 4.08 (2H, m, H-23), 2.76 (1H, br s, H-18), 1.54 (3H, s, $\left.\mathrm{CH}_{3}-25\right), 1.25$ (3H, s, $\left.\mathrm{CH}_{3}-27\right), 0.97\left(3 \mathrm{H}, \mathrm{s}, \mathrm{CH}_{3}-29\right), 0.93\left(3 \mathrm{H}, \mathrm{d}, J=6.64 \mathrm{~Hz}, \mathrm{CH}_{3}-30\right), 0.92(3 \mathrm{H}, \mathrm{s}$, $\left.\mathrm{CH}_{3}-26\right), 0.88$ (3H, s, $\left.\mathrm{CH}_{3}-24\right)$; ${ }^{13} \mathrm{C}-\mathrm{NMR} \delta: 178.3$ (C-28), 139.7 (C-13), 129.0 (C-12), 73.7 (C-3), 73.0 (C-19), 68.2 (C-23), 54.9 (C-18), 48.8 (C-5), 48.3 (C-9), 47.9 (C-17), 43.0 (C-20), 42.3 (C-14), 42.2 (C-8), 40.6 (C-1), 39.2 (C-4), 39.0 (C-22), 37.3 (C-10), 33.4 (C-7), 29.1 (C-15), 27.8 (C-21), 27.2 (C-29), 27.2 (C-2), 26.4 (C-16), 24.7 (C-27), 24.3 (C-11), 18.9 (C-6), 17.1 (C-25), 16.8 (C-26), 16.1 (C-30), 13.2 (C-24), 174.5 (C-1"'), 63.5 (C-3"'), 56.6 (C-2"'). ESI-MS m/z: 576.4 [M+H] $]^{+}$HR-ESI-MS found: 576.3911 . calcd: 576.3900 for $\mathrm{C}_{33} \mathrm{H}_{54} \mathrm{NO}_{7}\left([\mathrm{M}+\mathrm{H}]^{+}\right)$.

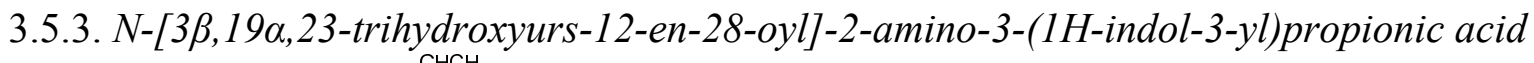 $\left(6 \mathbf{b}, \mathrm{C}_{41} \mathrm{H}_{58} \mathrm{~N}_{2} \mathrm{O}_{6}, \mathrm{R}_{1}={ }^{\mathrm{CHCH}_{2}}\right.$}

White powder, yield 88.2\%, mp $257 \sim 258^{\circ} \mathrm{C}$; IR $(\mathrm{KBr}) \mathrm{cm}^{-1}: 3463,3411,2930,2879,2862,1728$, 1658, 1499, 1457, 1444, 1387, 1357, 1242, 1042 and 743; ${ }^{1} \mathrm{H}-\mathrm{NMR} \delta: 11.9$ (1H, s, indole-NH), 7.98 (1H, brs, amide-NH), 7.48 (1H, brs, H-4"''), 7.46 (1H, s, H-2"''), 7.35 (1H, m, H-7"''), 7.16 (2H, m, H-5"'" and H-6"'), 5.33 (1H, m, H-12), 5.11 (1H, m, H-2"'), 4.91 (1H, m, H-3), 3.90 (2H, m, H-23), 3.64 (3H, s, 1'"--OCH $), 3.35$ (2H, m, H-3"'), 2.77 (1H, br s, H-18), $1.96\left(3 \mathrm{H}, \mathrm{s}, \mathrm{CH}_{3}-2^{\prime}\right), 1.85$ (3H, s, $\left.\mathrm{CH}_{3}-2^{\prime \prime}\right)$, $1.50\left(3 \mathrm{H}, \mathrm{s}, \mathrm{CH}_{3}-25\right), 1.20\left(3 \mathrm{H}, \mathrm{s}, \mathrm{CH}_{3}-27\right), 0.91\left(3 \mathrm{H}, \mathrm{d}, J=6.64 \mathrm{~Hz}, \mathrm{CH}_{3}-30\right), 0.75\left(3 \mathrm{H}, \mathrm{s}, \mathrm{CH}_{3}-29\right)$, $0.70\left(3 \mathrm{H}, \mathrm{s}, \mathrm{CH}_{3}-26\right), 0.29$ (3H, s, $\left.\mathrm{CH}_{3}-24\right) ;{ }^{13} \mathrm{C}-\mathrm{NMR} \delta: 178.2$ (C-28), 139.7 (C-13), 137.8 (C-9'"'), 
129.0 (C-8"'"), 128.7 (C-12), 1124.6 (C-2"'"), 121.9 (C-6"''), 119.6 (C-5"'"), 119.4 (C-7"'"), 112.3 (C-4"'"), 111.6 (C-3"'"), 73.9 (C-3), 73.0 (C-19), 68.3 (C-23), 54.6 (C-18), 48.8 (C-5), 48.0 (C-9), 47.9 (C-17), 43.0 (C-20), 42.3 (C-14), 42.1 (C-8), 40.4 (C-1), 39.0 (C-4), 38.9 (C-22), 37.3 (C-10), 33.1 (C-7), 28.8 (C-15), 27.8 (C-21), 27.2 (C-29), 27.2 (C-2), 26.4 (C-16), 24.7 (C-27), 24.2 (C-11), 18.9 (C-6), 16.9 (C-25), 16.7 (C-26), 16.1 (C-30), 13.2 (C-24), 175.8 (C-1"'), 28.1 (C-3"'), 55.0 (C-2"'). ESI-MS m/z: $675.3[\mathrm{M}+\mathrm{H}]^{+}$. HR-ESI-MS found: 675.4295. calcd: 675.4373 for $\mathrm{C}_{41} \mathrm{H}_{59} \mathrm{~N}_{2} \mathrm{O}_{6}\left([\mathrm{M}+\mathrm{H}]^{+}\right)$.

3.5.4. $N$-[3 $\beta, 19 \alpha$,23-trihydroxy urs-12-en-28-oyl]-2-amino-3-phenylpropionic acid $\left(7 \mathbf{b}, \mathrm{C}_{39} \mathrm{H}_{57} \mathrm{NO}_{6}\right.$, $\left.\mathrm{R}_{1}=\mathrm{CHCH}_{2}\right)$

White powder, yield 90.1\%, mp $219 \sim 221{ }^{\circ} \mathrm{C}$; IR $(\mathrm{KBr}) \mathrm{cm}^{-1}: 3195,3448,3393,2978,2931$, 28710, 1753, 1667, 1499, 1456, 1369, 1046 and 705; ${ }^{1} \mathrm{H}-\mathrm{NMR} \delta: 7.74$ (1H, brs, -NH), 7.42 (2H, m, H-2"'' and 6"''), 7.27 (2H, m, H-3"'' and 5"''), 7.17 (1H, m, H-4"''), 5.46 (1H, m, H-12), 5.12 (1H, m, H-2"'), 4.98 (1H, m, H-3), 4.05 (2H, m, H-23), 3.55 (2H, m, H-3"'), 2.65 (1H, br s, H-18) 1.52 (3H, s, $\left.\mathrm{CH}_{3}-25\right)$, $1.20\left(3 \mathrm{H}, \mathrm{s}, \mathrm{CH}_{3}-27\right), 0.97\left(3 \mathrm{H}, \mathrm{s}, \mathrm{CH}_{3}-29\right), 0.93\left(3 \mathrm{H}, \mathrm{d}, J=6.64 \mathrm{~Hz}, \mathrm{CH}_{3}-30\right), 0.86\left(3 \mathrm{H}, \mathrm{s}, \mathrm{CH}_{3}-26\right)$, $0.73\left(3 \mathrm{H}, \mathrm{s}, \mathrm{CH}_{3}-24\right) ;{ }^{13} \mathrm{C}-\mathrm{NMR} \delta: 178.3$ (C-28), 139.7 (C-13), 138.8 (C-1"''), 130.3 (C-2"'" and 6"''), 128.9 (C-3"' and 5"''), 128.8 (C-12), 127.2 (C-4"'"), 73.8 (C-3), 73.0 (C-19), 68.3 (C-23), 54.7 (C-18), 48.8 (C-5), 48.2 (C-9), 48.0 (C-17), 43.0 (C-20), 42.4 (C-14), 42.2 (C-8), 40.5 (C-1), 39.0 (C-4), 38.8 (C-22), 38.3 (C-10), 37.3 (C-7), 33.1 (C-15), 27.8 (C-21), 27.2 (C-29), 27.2 (C-2), 26.4 (C-16), 24.7 (C-27), 24.2 (C-11), 18.8 (C-6), 16.9 (C-25), 16.9 (C-26), 16.1 (C-30), 13.2 (C-24), 175.2 (C-1'"'), 28.9 (C-3"'), 55.4 (C-2"'). ESI-MS m/z: 636.3 [M+H] ${ }^{+}$. HR-ESI-MS found: 636.4221. calcd: 636.4259 for $\mathrm{C}_{39} \mathrm{H}_{58} \mathrm{NO}_{6}\left([\mathrm{M}+\mathrm{H}]^{+}\right)$.

\subsection{In Vitro Anti-tumor Assays}

Aliquots $(200 \mu \mathrm{L})$ of $5 \times 10^{3}$ cells per $\mathrm{mL}$ of A375, HepG2 and NCI-H446 cells were seeded in 96 well flat-bottomed plates in DMEM medium containing 10\% FBS and a penicillin-streptomycin mixture at $37{ }^{\circ} \mathrm{C}$ in a humidified atmosphere of $5 \% \mathrm{CO}_{2}$. The test drugs were dissolved in DMSO. The incubation medium was replaced with each test medium giving a final concentration of 5-40 $\mu \mathrm{mol} / \mathrm{L}$ of test compounds and no drug in $2 \mu \mathrm{L}$ DMSO over $24 \mathrm{~h}$. The ability of the drug to inhibit cellular growth was determined by performing the MTT assay. Each experiment was performed in six wells, and all the experiments involving a control (DMSO only). The drug treatments were performed separately three times. All data are presented as mean \pm standard deviations (S.D.). Statistical significance of the differences between groups was assessed by Student's $t$-test.

\section{Conclusions}

To date, though RA has been reported to show cytotoxicity, there are no reports on chemical modification of RA and the bioactivity of its derivatives. In this work, based on our previous investigation of RA, eight novel amino acid derivatives of RA were synthesized and their anti-tumor activities were tested in vitro for the first time. These eight compounds showed different cytotoxicities on the three tested tumor cell lines. Especially, compound 5a possesses better activity than RA, with 2.76-, 2.14-, 2.96-fold more potent activities than RA, respectively. The cytotoxicity of compound 5a 
was more sensitive than $\mathbf{R A}$, with an $\mathrm{IC}_{50}$ value of less than $10 \mu \mathrm{M}$ on all three cell lines. Compound 6b showed equivalent activity to RA on the HepG2 and NCI-H446 cell lines, and higher cytotoxicity on the A375 cell line. Compounds 5a and $\mathbf{6 b}$ may thus serve as potential lead compounds for the development of new anticancer drugs. More derivative synthesis and further biological evaluations are currently in progress and will be reported in due course.

\section{Acknowledgements}

This work was supported by Jilin Science \& Technology Development Plan (SC201102129 and 20116045). We also thank Christopher Beneduce and Zhaohui Sunny Zhou at the Department of Chemistry and Chemical Biology, Northeastern University, Boston for editing the manuscript and their suggestions.

\section{References and Notes}

1. Sun, H.; Zhang, X.Q.; Cai, Y.; Han, W.L.; Wang, Y.; Ye, W.C. Study on chemical constituents of Ilex rotunda Thunb. Chem. Indus. Forest Prod. 2009, 295, 111-114. In Chinese.

2. Xie, J.B.; Bi, Z.M.; Li, P. HPLC-ELSD determination of triterpenoids and triterpenoid saponins in Ilex pupurea leaves. Acta Pharmaceu. Sin. 2003, 38, 534-536. In Chinese.

3. Liao, L.P.; Bi, Z.M.; Li, P.; Xie, J.B.; Zhang, T.D. Triterpenoids from leaves of Ilex purpurea. Chin. J. Nat. Med. 2005, 3, 344-346. In Chinese.

4. Haraguchi, H.; Kataoka, S.; Okamoto, S.; Hanafi, M.; Shibata, K. Antimicrobial triterpenes from Ilex integra and the mechanism of antifungal action. Phytother. Res. 1999, 13, 151-156.

5. Zhao, W.M.; Wolfender, J.L.; Hostettmann, K.; Cheng, K.F.; Xu, R.S.; Qin, G.W. Triterpenes and triterpenoid saponins from Mussaenda pubescens. Phytochemistry 1997, 45, 1073-1078.

6. Bhattacharyya, J.; Almeida, M.D. Isolation of the constituents of the root-bark of Guettarda platypoda. J. Nat. Prod. 1985, 48, 148.

7. Saimaru, H.; Orihara, Y.; Tahsakul, P.; Kang, Y.H.; Shibuya, M.; Ebizuka, Y. Production of triterpene acids by cell suspension cultures of Olea europaea. Chem. Pharm. Bull. 2007, 55, 784-788.

8. Li, Z.H.; Zhang, H.S.; Xu, F.L.; Wu, Z.Y. Triterpene acids from the leaves of Planchonella duclitan (Blanco) Bakhuizan. J. Chin. Chem. Soc. 2005, 52, 1275-1280.

9. Zhao, Q.C.; Nan, M.L.; He, Y.F.; Chen, S.W. Application of Rotundic Acid in the Cardiovascular Disease Prevention. CHN 201010204596.9, 2010. In Chinese.

10. $\mathrm{Xu}, \mathrm{R}$. Studied on the Chemical Components and Antitumor Activity of Ilex rotunda Thunb. Ph.D. Thesis, Guangzhou University of Chinese Medicine, 2009. In Chinese.

11. Zhao, Q.C.; Nan, M.L.; He, Y.F.; Chen, S.W. Application of Rotundic Acid in the Preparation of Lipid-lowering Drugs.CHN 201010204607.3, 2010. In Chinese.

12. He, Y.F.; Zhao, Q.C.; Nan, M.L.; Wang, H.L.; Ma, J.S.; Zhao, Y.W.; Wang, L.P. Application of Rotundic Acid and Its Derivatives in the Preparation of Anticancer Drugs. CHN 201010607515.x, 2010. In Chinese.

13. Nan, M.L.; Zhao, Q.C.; He, Y.F.; Chen, S.W.; Zhao, Y.W.; Wang, L.P. Pharmaceutical Compositions from Ilex rotunda Thunb. and Its Application. CHN 201010607550.1, 2010. In Chinese. 
14. Zhao, Q.C.; He, Y.F.; Nan, M.L.; Chen, S.W.; Zhao, Y.W.; Wang, L.P. Synthesis Method of Rotundic Acid Derivatives and Their Application in the Preparation of Cardiovascular Disease Prevention Drugs. CHN 20110030007.4, 2011. In Chinese.

15. He, Y.F.; Nan, M.L.; Zhao, Q.C.; Zhao, Y.W.; Yue, F.G. Application of Amino Acid Modified Rotundic Acid Derivatives in the Preparation of Anticancer Drugs. CHN 201110351365.5, 2011. In Chinese.

16. Lin, F.P.; Shao, J.W.; Du, H.D.; Dai, Y.C.; Wang, T. Synthesis, characterization and anti-tumor activity of ursolic acid derivatives. Chin. J. Appl. Chem.2010, 27, 893-898. In Chinese.

17. Liu, D.; Meng, Y.Q.; Zhao, J.; Chen, L.G. Synthesis and anti-tumor activity of novel amide derivatives of ursolic acid. Chem. Res. Chin. Univ. 2008, 24, 42-46.

18. Meng, Y.Q.; Liu, D.; Cai, L.L.; Chen, H.; Cao, B.; Wang, Y.Z. The synthesis of ursolic acid derivatives with cytotoxic activity and the investigation of their preliminary mechanism of action. Bioorg. Med. Chem. 2009, 17, 848-854.

19. Shibata, S.; Takahashi, K.; Yano, S.; Harada, M.; Saito, H.; Tamura, Y.; Kumagai, A.; Hirabayashi, K.; Yamamoto, M.; Nagata, N. Chemical modification of glycyrrhetinic acid in relation to the biological activities. Chem. Pharm. Bull. 1987, 35, 1910-1918.

20. Jain, S.M.; Atal, C.K. Synthesis of amino derivatives of ursolic acid. Indian J. Chem. 1986, 25B, 427-428.

21. Saier, M.H.; Daniels, G.A.; Boerner, P.; Lin, J. Neutral amino acid transport systems in animal cells: Potential targets of oncogene action and regulators of cellular growth. J. Membr. Biol. 1988, 104, 1-20.

22. Eagle, H.; Oyama, V.; Levy, M. Amino acid requirements of normal and malignant human cells in tissue culture. Arch. Biochem. Biophys. 1957, 67, 432-446.

23. Xu, P.; Fu, Y.Q.; Zou, X.M. Compounds with the Function of Proteasome Inhibition and Their Preparation Methods and Applications. CHN 200610012149.7, 2006. In Chinese.

24. Ohrai, K.; Adachi, M.; Toyama, K. Preparation of $\alpha$-Amino Acid Derivatives as Dual Iohibitors of Aurora Kinase and Cyclin-Dependent Kinase (CDK). JPN 2008001883, 2008.

25. Berst, F.; Grosche, P.; Janser, P.; Janwer, P.; Zecri, F.; Bloobuck, B. Preparation of N-biaryl (hetero) arylsulphonamide amino acid derivatives as sphingosine-1-phosphate recepctor type 1 antagonists useful in the treatment of diseases mediated by lymphocytes interactions. Swiss Patent Application 2008028937, 2008.

26. Kim, N.C.; Desjardins, A.E.; Wu, C.D.; Kinghorn, A.D. Activity of triterpenoid glycosides from the root bark of Mussaenda macrophylla against two oral pathogens. J. Nat. Prod. 1999, 62, 1379-1384.

27. Nakatani, M.; Hatanaka, S.; Komura, H.; Kubota, T.; Hase, T. The structure of rotungenoside, a new bitter triterpene glucoside from Ilex rotunda. Bull. Chem. Soc. Jpn. 1989, 62, 469-473.

28. Zhang, A.L.; Ye, Q.; Li, B.G.; Qi, H.Y.; Zhang, G.L. Phenolic and triterpene glycosides from the stems of Ilex litseaefolia. J. Nat. Prod. 2005, 68, 1531-1535.

29. Xie, G.B.; Zhou, S.X.; Lu, Y.N.; Lei, L.D.; Tu, P.F. Triterpenoid glycosidesfrom the leaves of Ilex pernyi. Chem. Pharm. Bull. 2009, 57, 520-524.

30. Zhuo, R.X.; Fan, C.L.; Zhao, R.L. Synthesis and antitumor activity of 5-fluorouracil containing amino acid derivatives. Chem. J. Chin. Univ. 1986, 7, 508-512. In Chinese. 
31. Zhao, R.L.; Fan, C.L.; Zhuo, R.X. Synthesis and antitumor activity of polyphosphoramides and polyphosphates containing amino acid, 5-fluorouracil and phosphonoformate or phosphonoacetate. Funct. Polym. 1989, 2, 223-230. In Chinese.

32. Zhuo, R.X.; Fan, C.L.; Zhuo, R.X. Synthesis and antitumor activity of polyphosphoramides and polyphosphates containing amino acid, 5-fluorouracil and nitrogen mustard. Funct. Polym. 1989, 2, 304-308. In Chinese.

33. Zhuo, R.X.; Fan, C.L.; Zhuo, R.X. Synthesis of amino acid 5-fluorouracil esters derivatives and study on their antitumor activity. Chem. J. Chin.Univ. 1989, 10, 605-608. In Chinese.

34. Zhuo, R.X.; Liu, G.W.; Peng, P.P. The synthesis and antitumor activity of 5-Fluorouracil- $N^{1}-$ carbonyl aminoacids and oligopeptides. Chem. J. Chin. Univ. 1991, 12, 555-559. In Chinese.

35. Sun, H.; Hu, C.; Fang, W.S. Synthesis, water solubility and antitumor activity of amino acid conjugates of 3-oxooleanolic acid. Chin. J. Med. Chem. 2008, 18, 11-15.

36. Mosmann, T. Rapid colorimetric assay for cellular growth and survival: Application to proliferation and cytotoxicity assays. J. Immunol. Methods. 1983, 139, 55-63.

Sample Availability: Samples of the compounds $\mathbf{R A}, \mathbf{4 a}-\mathbf{7 a}, \mathbf{4 b}-\mathbf{7 b}$ are available from the authors.

(C) 2012 by the authors; licensee MDPI, Basel, Switzerland. This article is an open access article distributed under the terms and conditions of the Creative Commons Attribution license (http://creativecommons.org/licenses/by/3.0/). 